\title{
QUALITY BY DESIGN: AN OVERVIEW OF THE BASIC CONCEPTS
}

Rohin Mhatre and Anurag S. Rathore

\subsection{INTRODUCTION}

The premise of Quality by Design (QbD) is that the quality of the pharmaceutical product should be based upon the understanding of the biology or the mechanism of action (MOA) and the safety of the molecule [1]. The manufacturing process should then be developed to meet the desired quality attributes of the molecule, hence the concept of "design" of the product quality versus "testing" the product quality. Although testing the product quality after manufacturing is an essential element of quality control, testing should be conducted to confirm the predesired product attributes and not to simply reveal the outcome of a manufacturing process. The ICH Q8 guideline provides an overview of some of the aspects of QbD [2]. The guideline clearly states that quality cannot be tested into products; that is, quality should be built in by design.

Although the task of designing a complex biological molecule such as a monoclonal antibody may seem daunting, the experience gained in the past roughly 30 years of the biotechnology industry history has laid the foundation for the QbD initiative [3,4]. The industry has come a long way in identifying and selecting viable drug candidates, in developing high-productivity cell culture processes, in designing purification processes that yield a high-purity product, and in analyzing the heterogeneity of complex 
biomolecules. As all these activities are the building blocks of QbD, the concept of QbD has in fact been practiced for the last few years and has in turn led to the development of highly efficacious biopharmaceuticals and robust manufacturing processes. The issuance of the ICH Q8 guideline was an attempt to formalize the QbD initiative and to allow manufacturing flexibility based on the manufacturer's intricate knowledge of the molecule and the manufacturing process. The concept of obtaining intricate knowledge of the molecule along with the manufacturing process and the resulting flexibility in manufacturing, the eventual goal of the QbD initiative, requires an understanding of the various elements of $\mathrm{QbD}$.

The two key components of QbD are [4]

1. The understanding of the critical quality attributes (CQAs) of a molecule. These are the attributes of the molecule that could potentially affect its safety and efficacy profile.

2. The design space of the process defined as the range of process inputs that help ensure the output of desired product quality.

An overview of these components is discussed further in this chapter and elsewhere in this book.

\subsection{CRITICAL QUALITY ATTRIBUTES}

The starting point of QbD is developing a good understanding of the molecule itself. Biomolecules are quite heterogeneous due to the various post-translational modifications that can occur and have been commonly observed. These modifications arise from the glycosylation, oxidation, deamidation, cleavage of labile sites, aggregation, and phosphorylation, to name a few. As many of these modifications could impact the safety and efficacy of the molecule, defining the appropriate CQAs of the molecule is an important starting point in the development cycle of a biopharmaceutical. Although the understanding of the CQAs evolves during the life cycle of the product, understanding the CQAs at an early stage of the development of the molecule is clearly desirable. Studies conducted during the early research stages of development of a potential biopharmaceutical may entail evaluating various forms of a particular biomolecule in animal studies. The outcomes of such studies help "design" a biomolecule with the desired quality attributes so as to be safe and highly efficacious.

Since the CQAs can impact the safety and clinical efficacy of a molecule, data gathered in animal studies, toxicological studies, and early human clinical trails become the starting point for defining the CQAs. On the basis of the safety and efficacy readout of a clinical trial, one can start to define the product profile of a molecule. The assumption is that if the CQAs of the molecule are similar to those used in preclinical and clinical trials, the safety and efficacy will be comparable as well. Furthermore, historical data from clinical trials of similar molecules can also provide valuable insight into the CQAs. Evaluation of the in vitro biological activity via bioassays, reflecting the mechanism of action, can provide a good assessment of how the various product attributes could 
potentially impact the in vivo activity of a molecule. The molecule can be altered by conducting stress studies to induce higher level of aggregation; oxidation, deamidation, and the glycosylation pattern can be varied as well. The impact of changes in the molecular structure on the biological activity can then be evaluated via various bioassays. This study is referred to as structure-activity relationship. The evaluation of in vitro activity is often the relatively easiest means of determining the CQAs. However, in vitro assessments can only provide an understanding of the potential changes in the activity of the molecule, and the correlation between this change in activity and the impact of efficacy in patients is often unclear. Further assessment of the molecule in animal studies to evaluate clearance, efficacy, and safety is often a good indicator of the behavior of the molecule in human trials and is a better tool for understanding the CQAs. Additional details of the determination of the CQA can be found in Chapter 4.

\subsection{AN OVERVIEW OF DESIGN SPACE}

After defining the CQAs, the next and more critical step is the development of a manufacturing process that will yield a product with the desired CQAs $[4,5]$. During the process development, several process parameters are routinely evaluated to assess how they could impact product quality [6]. The design space for the process eventually evolves from such a study. For example, during the cell culture development, ranges for process inputs such as temperature, $\mathrm{pH}$, and the feed timing can be evaluated to determine if operating within a certain range of temperature and $\mathrm{pH}$ has an impact on product quality. The design of experiments (DOE) is conducted in a manner so as to evaluate the impact of the multiple variables (multivariate) and also to understand if and how changes in one or more of the process inputs have an effect on the product quality and/or if a process input is independent of changes in other inputs.

The design space (process range) is then established for each of the above process inputs. This can be further explained using an example of a design space for a purification column. If a column used to purify a protein is expected to reduce the level of protein aggregate to $2 \%$, the various column operating parameters such as flow rate, $\mathrm{pH}$ and strength of the buffer, load volume, and so on are evaluated such that operating within a certain range of these parameters yields an aggregate level of less than or equal to $2 \%$. If it turns out that $\mathrm{pH}$ above 6 or below 4 results in aggregate levels above $2 \%$, then the design space for the $\mathrm{pH}$ of the buffer is defined as between 4 and 6 . One can similarly envision a design space for the flow rate and other inputs for the particular purification step. Eventually, the entire production process for a molecule will have a defined design space, and operating within that design space should lead to a product of acceptable quality. Operating beyond the design space of a particular process input may result in an unacceptable product quality.

Since the production process for a biomolecule entails multiple steps starting from the cell culture process to the final purification and eventually to the formulation and fill in the desired container, the development of a design space for a particular step is not usually independent of other steps in the production process. Since the output of one step becomes the input for the next step, the development of the design space for a process 
should be evaluated in a holistic manner. One such approach may be to determine the desired product quality from the final process step and to work backward in the process to ensure that each step of the process delivers the required product quality needed for the next step to meet the quality target of the final step. To provide an example of this approach, we can revisit the above example of a desired level of $2 \%$ aggregate in the final drug product. In this particular case, design space should be developed for all parameters of the production process that can potentially impact the level of aggregate in the final drug product. The maximum level of aggregate resulting from each of the steps in the process does not need to be less than $2 \%$, particularly steps that are upstream in the process such as the protein A purification, the first columns used in the purification of an antibody. The development of the design space including the design of experiments is discussed in detail in Chapters 5-7.

\subsection{RAW MATERIALS AND THEIR IMPACT ON QbD}

In addition to the design space and CQAs, other factors also play an important role in implementing $\mathrm{QbD}$, and raw material is one such factor. Cell culture processes used to make recombinant proteins use complex growth media such as hydrosylates and also feeds such as vitamins for the cell. The understanding of how the various components of these complex raw materials affect the productivity of the cells and the quality of the product is not a trivial task. It requires a thorough analysis and quantitation of the various components of the raw material. Raw material analysis and correlation between raw material components and the productivity of cells and product quality is an area that has not been sufficiently explored by the biotechnology industry. However, the evolution of instruments such as high-resolution nuclear magnetic resonance spectroscopy, near infrared spectroscopy, and mass spectrometry has provided an opportunity to analyze complex mixtures of raw materials. In addition, the availability of sophisticated statistical tools for deconvolution and pattern matching of complex data sets has further refined the approach to analyzing raw materials. Once the correlation between the critical components of the growth or feed media and the performance of cells is understood, the ultimate goal of raw material analysis in the context of QbD would be to fortify the media as needed with the relevant component so as to ensure the desired productivity and product quality. Further details of analysis of complex raw materials are provided in Chapter 11.

\subsection{PROCESS ANALYTICAL TECHNOLOGY}

Since one of the goals of $\mathrm{QbD}$ is to maintain control of the process to achieve the desired product attributes, process analytical technology (PAT) is an important tool for QbD. PAT entails analysis of product quality attributes during the various stages of the manufacturing process of a biomolecule. The analysis is often conducted online using either probes inserted into the bioreactor to monitor critical components such as the cell density or sterile sampling devices to divert the stream from a purification column to assess the 
product purity [7]. In either case, the online analysis enables operators on the manufacturing floor to make real-time adjustments to the process parameters so as to obtain the desired product profile at every stage of the manufacturing process. For example, a PAT tool to monitor a purification column would entail periodically sampling the elution stream from the column via a sampling device and diverting the sample to an online HPLC system [8]. The results of the online HPLC analysis, indicative of the product purity, would be used to determine the eluate volume that should be collected. In this particular example, the fraction of the eluate of purity below a predetermined criterion would provide a trigger to stop collection of the eluate and to divert the elution stream to waste. The advantage of such a PAT tool would be that the collection of the column eluate would be based on the required product purity and would help to ensure a consistent product quality for every production batch of the biomolecule [8]. Further applications of PAT can be found in Chapters 12 and 13.

\subsection{THE UTILITY OF DESIGN SPACE AND QbD}

Prior to development of the design space, the questions to ask are the following: How would the design space be used? What is the advantage for a company of developing a design space for any of its products? What would be the driver for regulatory agencies to promote the concept of design space and QbD?

As seen in Fig. 1.1, limits that establish the acceptable variability in product quality and process performance attributes would also serve as the process validation acceptance criteria $[4,5]$. After the design space has been established, the regulatory filing would include the acceptable ranges for all key and critical operating parameters (i.e., design space) in addition to a more restricted operating space typically described for pharmaceutical products. After approval, CQAs would be monitored to ensure that the process is performing within the defined acceptable variability that served as the basis for the filed design space. The primary benefit of an expanded design space would be a more flexible approach by regulatory agencies. Process changes are often driven by changes in the manufacturing equipment and raw materials, to name a few. At present, changes in the process require formal filings and approvals from regulatory agencies and often require a significant commitment of both time and resources for the industry and the regulatory agencies. The outcome of the design space development (as stated in the ICH Q8 guideline) would be that upon the approval of the design space for a particular product by a regulatory agency, process changes within the design space would not require additional regulatory filing and approval. This shift in the paradigm of using enhanced process knowledge to enable process changes with a limited burden of regulatory approval is clearly beneficial to both the manufacturer and the regulatory agencies. Chapter 2 further reviews the regulatory relief and implications of the QbD initiative.

Process improvements during the product life cycle with regard to process consistency and throughput could take place with reduced postapproval submissions. As manufacturing experience grows and opportunities for process improvement are identified, the operating space could be revised within the design space without the need 


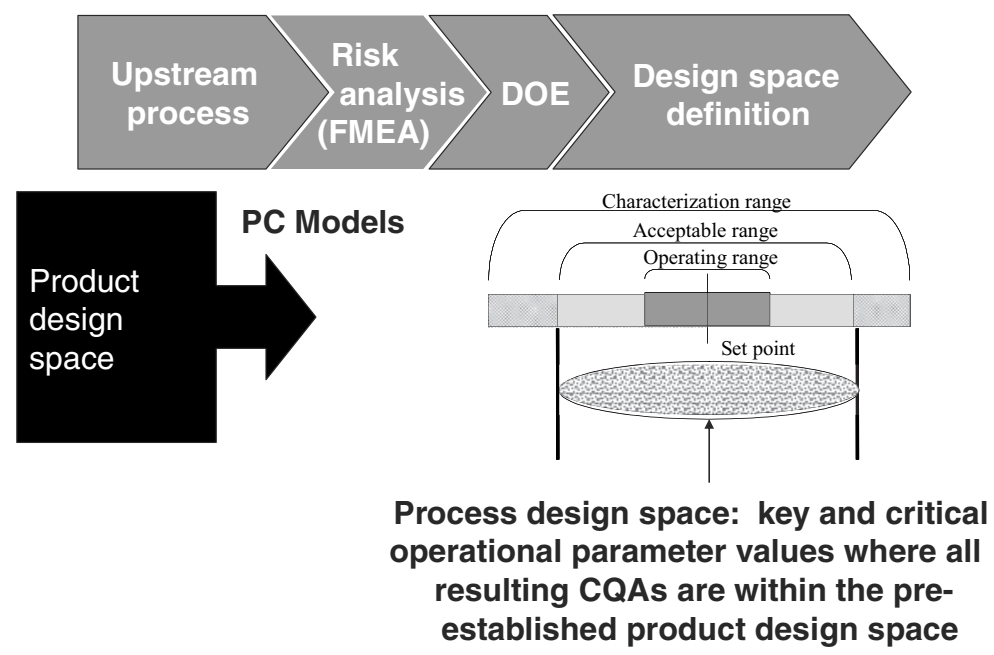

Figure 1.1. Illustration of the creation of process design space from process characterization studies and its relationship with the characterized and operating spaces. The operating range denotes the range in the manufacturing procedures and the characterization range is the range examined during process characterization. The acceptable range is the output of the characterization studies and defines the process design space. Adapted from Ref. [5], by permission of Advanstar Communications.

for postapproval submission. This is illustrated in Fig. 1.2, which shows that if the process creeps outside the design space, process changes may be required to be made and may require process characterization, validation, and filing of the changes to the approved process design space.

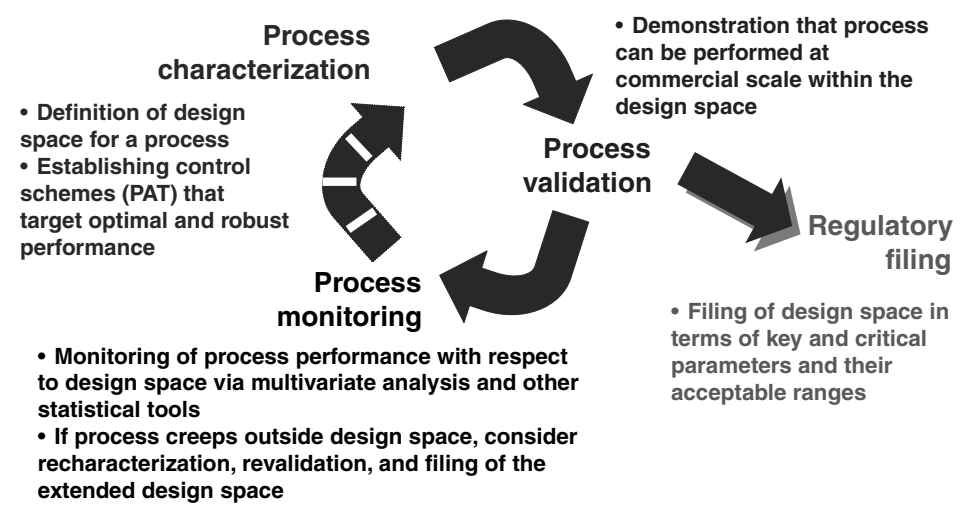

Figure 1.2. Application of the design space concept in process characterization, validation, monitoring, and regulatory filing. Adapted from Ref. [5], by permission of Advanstar Communications. 


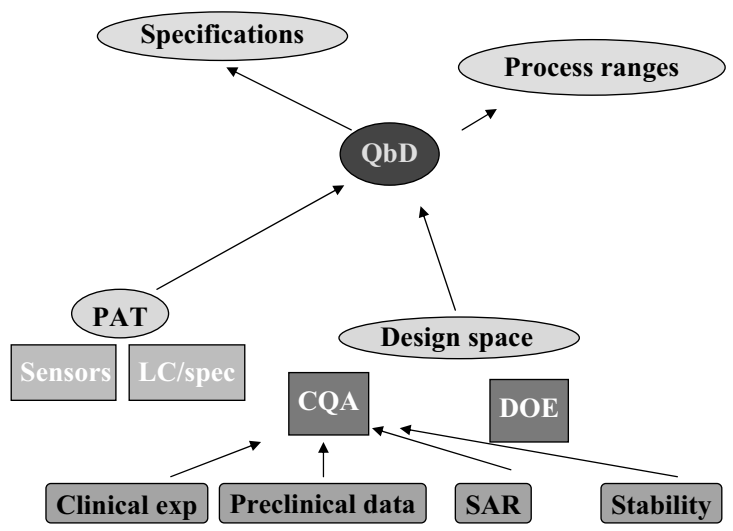

Figure 1.3. The various elements of QbD. The boxes in the bottom row show all the relevant information that is used to develop the critical quality attributes. The CQA and DOE data are then used to develop the design space. The design space and PAT tools help establish QbD.

\subsection{CONCLUSIONS}

Figure 1.3 depicts the various components of $\mathrm{QbD}$ discussed above and the correlation between the various components. As shown in the figure, the outcome of the QbD exercise is the establishment of the design space for the process and the operating ranges (ORs) that help achieve the desired product quality. As mentioned earlier, the reader is referred to the various sections of the book to gain further understanding of the various aspects of QbD. The editors hope that this book will help establish a good framework for any researcher to build Quality by Design into a manufacturing process for a biomolecule.

\section{REFERENCES}

[1] PAT Guidance for Industry: A Framework for Innovative Pharmaceutical Development, Manufacturing and Quality Assurance. U.S. Department of Health and Human Services, Food and Drug Administration (FDA), Center for Drug Evaluation and Research (CDER), Center for Veterinary Medicine (CVM), Office of Regulatory Affairs (ORA), September 2004.

[2] Guidance for Industry: Q8 Pharmaceutical Development. U.S. Department of Health and Human Service, Food and Drug Administration (FDA), May 2006.

[3] Kozlowski S, Swann P. Current and future issues in the manufacturing and development of monoclonal antibodies. Adv Drug Deliv Rev 2006;58:707-722.

[4] Rathore AS, Winkle H. Quality by Design for Pharmaceuticals: Regulatary Perspective and Approach. Nature Biotechnology 2009;27:26-34.

[5] Rathore AS, Branning R, Cecchini D. Design space for biotech products. BioPharm Int 2007;36-40. 
[6] Harms J, Wang X, Kim T, Yang J, Rathore AS. Defining design space for biotech products: case study of Pichia pastoris fermentation. Biotechnol Prog 2008;24(3):655-662.

[7] Munson J, Stanfiled CF, Gujral B. A review of process analytical technology (PAT) in the U.S. pharmaceutical industry. Curr Pharm Anal 2006;2:405-414.

[8] Rathore AS, Yu M, Yeboah S, Sharma A. Case study and application of process analytical technology (PAT) towards bioprocessing: use of on-line high performance liquid chromatography (HPLC) for making real time pooling decisions for process chromatography. Biotechnol Bioeng 2008;100:306-316. 